\title{
GALAXY FORMATION AND EVOLUTION (DISCUSSION SESSION)
}

\author{
Gustavo Bruzual \\ Centro de Investigaciones de Astronomia (CIDA) \\ Apartado Postal 264 \\ Merida 5101-A \\ Venezuela
}

\section{DISCUSSION}

The discussion on this topic was a lively one. The interest of the audience centered mainly on the papers about The Formation of Disk Galaxies (K. Freeman), The Formation of Elliptical Galaxies (B. Jones), The Spatial Clustering of QSO's (T. Shanks) programmed for the Thursday afternoon session, and on very brief ( $<5$ minutes) contributions on $A$ Possible Connection between the X-ray Background and the Large Scale Structure of the Universe (E. Daly), Constraints on Galaxy Formation from the Theory of the Inter Galactic Medium (P. Shapiro), Origin of the Hubble Sequence (G. Efstathiou), Appearance of Primeval Galaxies (S. White), and Hot Dark Matter and Cosmic Strings ( $R$. Brandenberger) that were scheduled for the following morning due to lack of time. G. Bruzual and G. Illingworth acted as discussion leaders.

Freeman argued in favor of a picture in which the thin disk of the Galaxy forms first. Then the halo forms by accretion of nearby metal poor satellites. The thick disk and central bulge will result from the heating of the stars in the thin disk by dynamical friction with the accreted material.

Ostriker pointed out that the main objection against the idea that this is the formation mechanism for all disk galaxies, is the well known observational fact that the brighter bulges have a higher metal content. This is not expected if the bulge is formed by heating disk stars of well defined metallicity.

Jones reviewed the different correlations existing among $\mathrm{E}$ galaxy properties. He strengthened the fact that even though $\mathrm{E}$ galaxies are found preferentially in rich clusters of galaxies, an E galaxy in the field or in a loose group is indistinguishable from one in a rich cluster. At the end of the discussion people seemed to agree on the idea that the similarity among the $\mathrm{E}$ galaxies may be just morphological. If their properties are studied in detail there may be differences from one to another galaxy.

In view of Freeman arguments, it was also clear that even though $\mathrm{E}$ galaxies resemble central bulges of spirals (in what sense, asked Ostriker), they may be formed according to different mechanisms. 
Shanks presented a statistical analysis of the spatial clustering of QSO's and galaxies. He detects at the 5 sigma level that QSO clustering is higher than galaxy clustering but not as much as Abell's clusters. QSO's seem to form in dense environments, but not as dense as Abell's clusters. He does not find any statistical evidence of clustering of galaxies in the 10 to $100 \mathrm{Mpc}$ scale. This result does not agree with the detection (by eye) of shells in the 3D distribution of galaxies reported by the CfA group. Shanks argued strongly in favor of using sound statistical techniques to establish the existence of these apparent shell shaped structures.

White reported his model (in collaboration with Baron) for the formation of (primeval) galaxies such as 3 C326.1 (observed by Spinrad, Djorgovski, and collaborators). The use of this model in the interpretation of this particular galaxy was subject to serious criticism by the audience.

M. Davis pointed out that 3C326.1 shows radio emission lobes, which argue in favor of the existence of an active nucleus. This object seems to be a well developed galaxy, and not precisely a primeval one. Davis also pointed out that there is a very large velocity difference (about $1000 \mathrm{~km} / \mathrm{s}$ ) from one end to the other of this object. It may not be bound.

On the same subject Gunn argued that $3 C 326.1$ is so extended at $z=$ 1.8 that it will never have the size of a large elliptical galaxy at $\mathbf{z}=0$.

Silk added that this galaxy is so blue at $z=1.8$ that in order for this population to reach the colors of $E$ galaxies at $z=0$, the value of $\mathrm{H}_{0}$ would have to be $20 \mathrm{~km} / \mathrm{s} / \mathrm{Mpc}$.

White claimed that this was not a fair comparison, because their model did not intend to explain the formation of $\mathbf{E}$ galaxies. He argued that one can still use the model to predict L L $\gamma_{\alpha}$ emission, and discard the small details and the comparison with this specific object.

The feeling among the audience was that galaxy formation models must be self consistent. These models must explain as many aspects of the problem as possible, not just isolated facts.

Because of lack of time no discussion was allowed after the oral presentations by Daly, Shapiro, Efstathiou, and Brandenberger. The reader is suggested to look for their papers in this volume. 